\title{
The London PCHF: A new Postgraduate Course on Heart Failure
}

\author{
Thomas F. Lüscher
}

\author{
Research, Education and Development, Royal Brompton and Harefield \\ Hospital Trust and Imperial College, London, United Kingdom
}

Reprinted in adapted form with permission from by the Zurich Heart House, Zurich, Switzerland

\section{The heart failure epidemic}

Heart failure is a true medical epidemic for several reasons. Indeed, as societies age in the Western world, with long-standing high blood pressure in many individuals and the high survival rate of myocardial infarction thanks to effective interventional techniques, the prevalence and incidence of heart failure has continuously increased. This is associated not only with an impaired quality of life of the patients, but also with considerable costs for the health care systems and the society at large.

The main causes of heart failure are longstanding hypertension, arrythmias, coronary artery disease, mutations in genes of the contractile apparatus and valvular heart disease (Figure 1).

\section{Increasing therapeutic options}

Although, initially, heart failure was a condition with very unfavourable outcomes, recent developments of the management of such patients has markedly increased their longevity and reduced hospitalisations. Thanks to this medical progress today, cardiologists have many therapeutic options at hand, such as life style changes and rehabilitation, drugs, revascularisation with percutaneous coronary intervention or bypass surgery and valvular interventions, pacemakers and implantable cardioverter defibrillators (ICD), as well as cardiac resynchronisation therapy, and finally left ventricular assist devices (Figure 2). For selected patients, even heart transplantation may be an option.

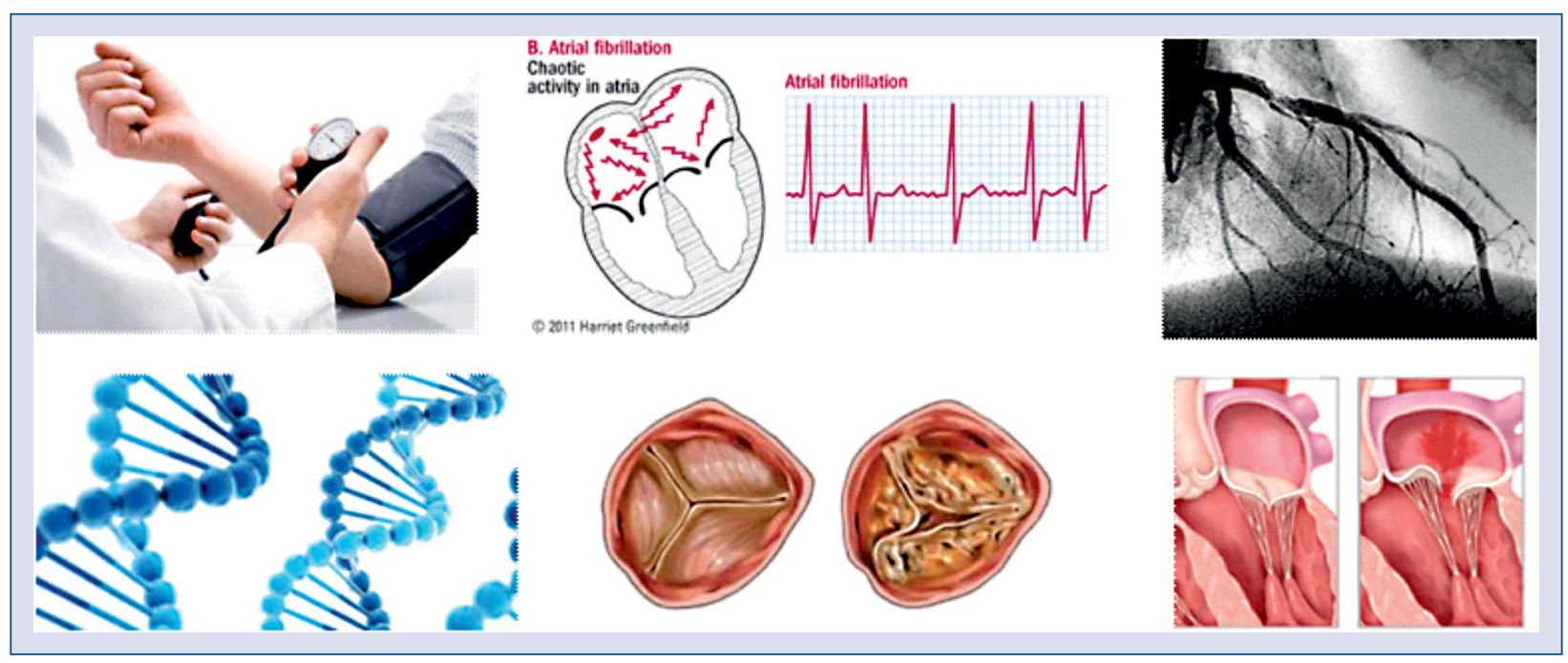

Figure 1. Most important causes of heart failure including hypertension, arrhythmias, coronary artery disease, genetics and valvular heart disease.

Address for correspondence: Thomas F. Lüscher, MD, FRCP, Professor of Cardiology Imperial College, Director of Research, Education and Development, Royal Brompton and Harefield Hospitals, Sydney Street, London SW3 6NP, United Kingdom, e-mail: cardio@tomluescher.ch 


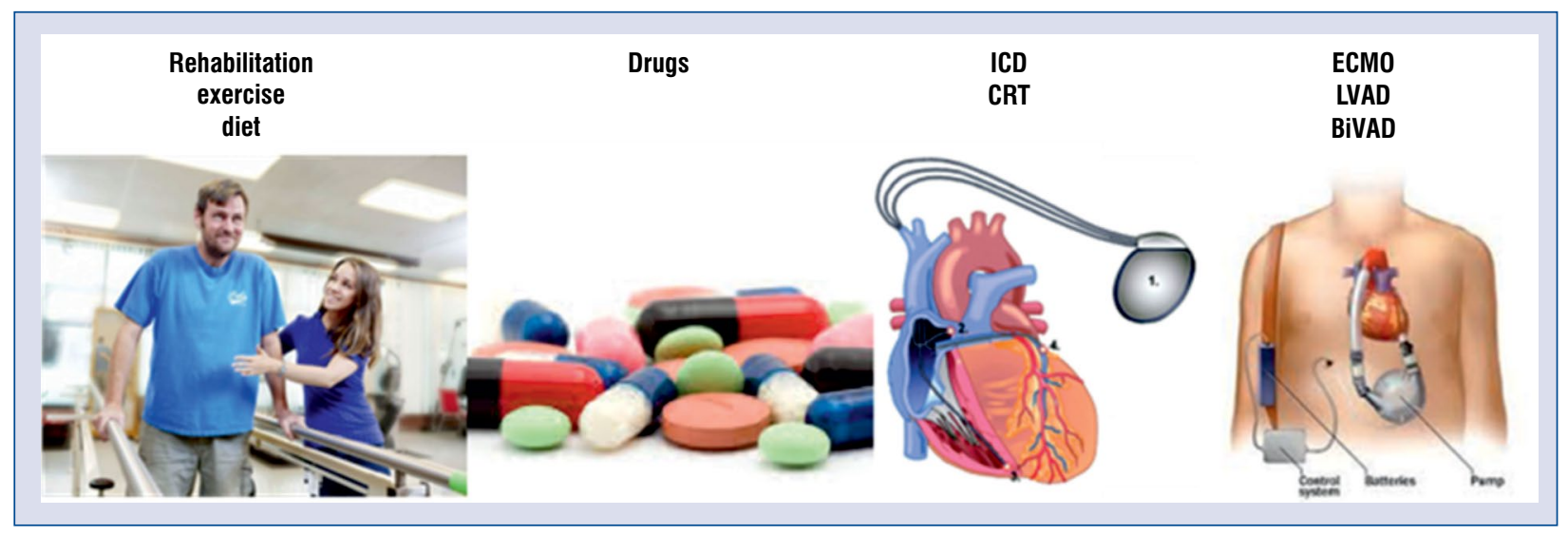

Figure 2. Therapeutic options in heart failure.

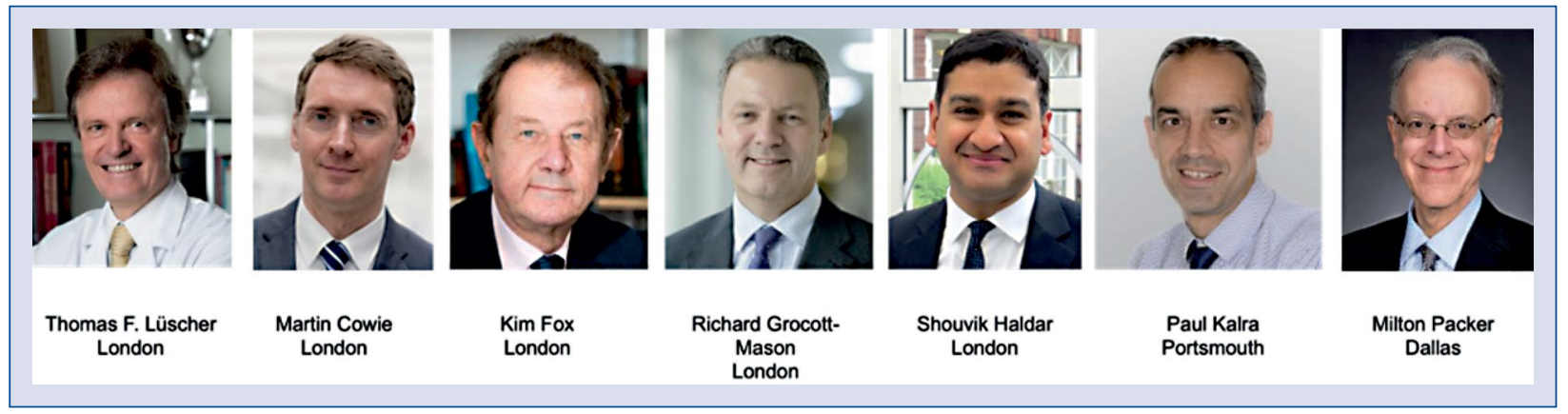

Figure 3. Directors of the London PCHF.

With these options the outcome of heart failure patients could be markedly improved both in reducing hospitalisations for heart failure and death.

Thus, heart failure doctors need an excellent training in all diagnostic and treatment modalities to properly take care of their patients.

\section{The London PCHF}

The new London Postgraduate Course in Heart Failure (THE LONDON PCHF), organised by the Royal Brompton and Harefield Hospitals and its Institute for Medicine and Science with support from the British Cardiovascular Society and the British Society for Heart Failure offers an educational programme at the highest academic and medical level. The experience and commitment of the Zurich Heart House and his educational team provides overall professional support for faculty and participants. The course directors are responsible for the content of the programme (Figure 3).

\section{Course participants}

The participants have been selected from close to 200 applicants by the Advisory Board of

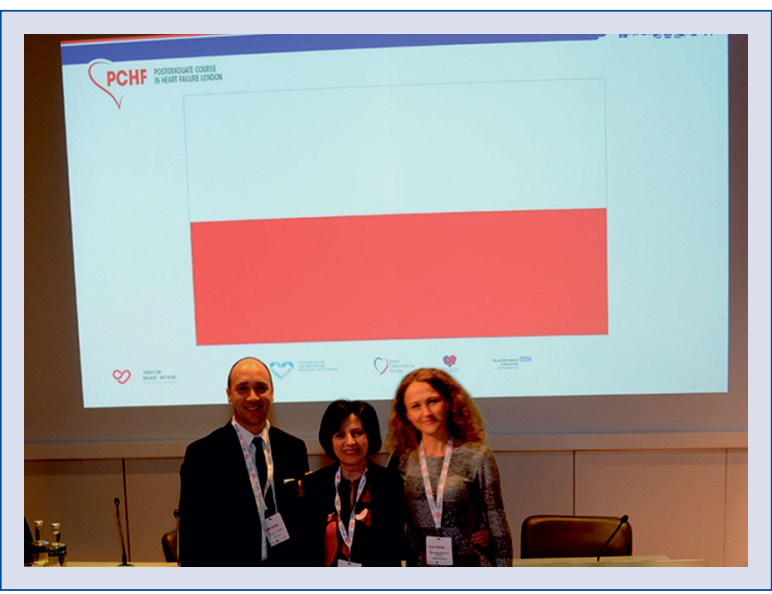

Figure 4. Participants from Poland (from left to right): Michal Bohdan, Malgorzata Lelonek, Maria Niespialowska-Steuden (Credit J. Lipton)

the London PCHF. Eventually, 64 participants from 32 countries mainly from Europe, but also from the far east, South Africa and the Americas have been accepted. Of note, half of the participants each are of female and male gender. The requirements for application included a board certificate in cardiology (or shortly before it), interest in heart failure, pub- 


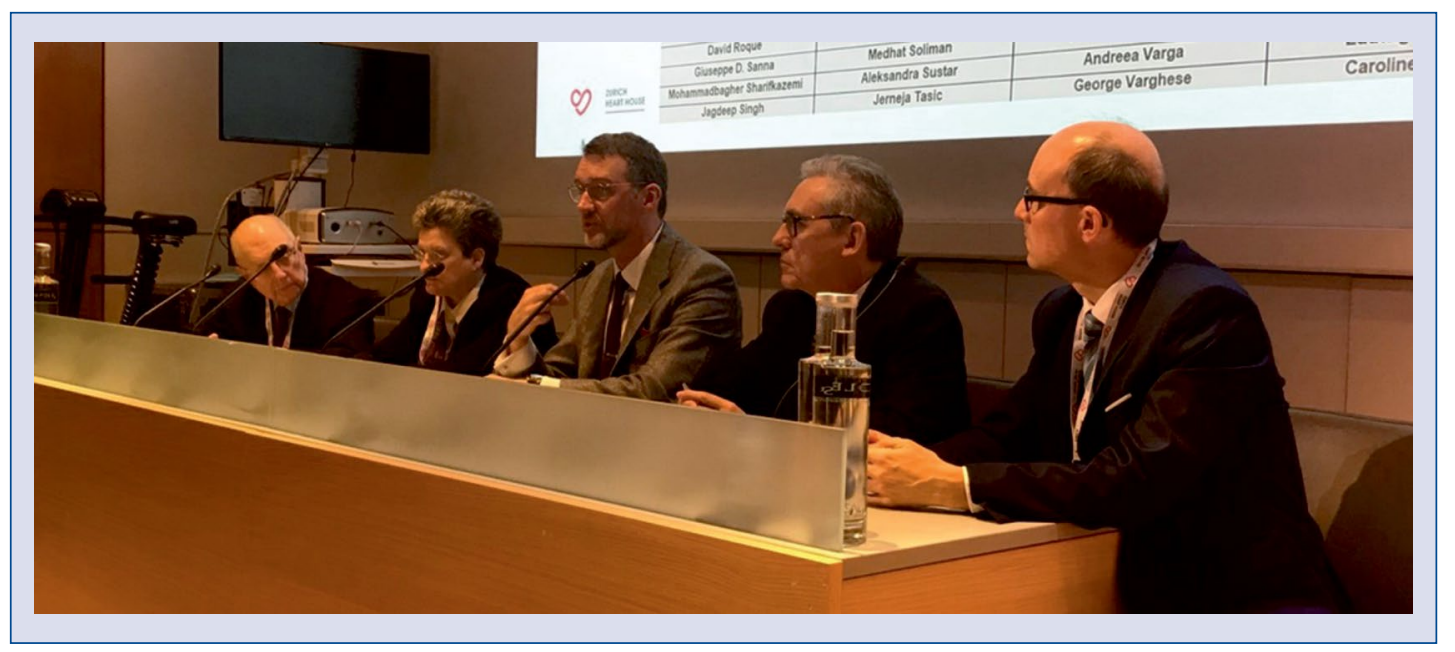

Figure 5. Opening Session of the London PCHF with Bertram Pitt, Teresa McDonagh, Martin Cowie, John Cleland and Christian Mueller.

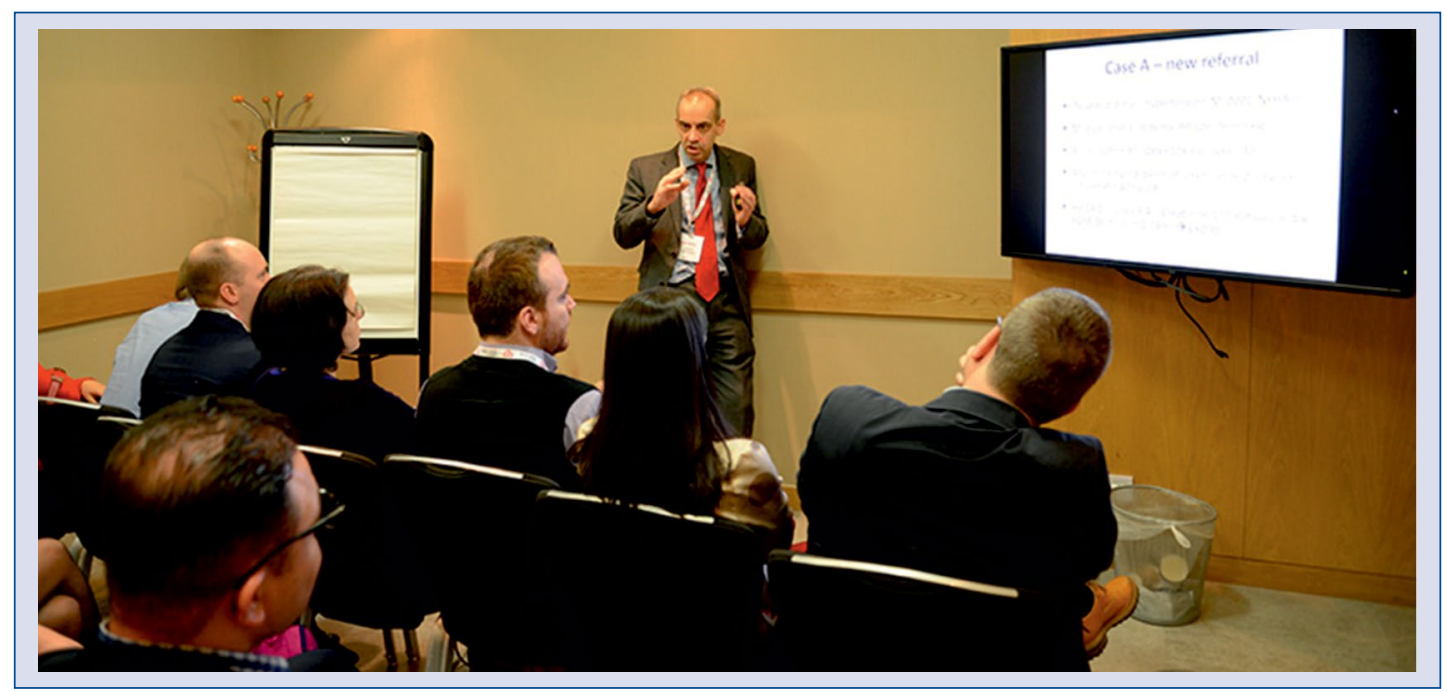

Figure 6. Paul Kalra discusses clinical cases in a breakout session (Credit J. Lipton).

lications on the topic and a recommendation letter by a supervisor. All 64 cardiologists participated in the first module that took place on January 16-19, 2019 at The Royal Society of Medicine, in Central London, as well as practical courses at the Royal Brompton and Harefield Hospitals, among them also three young cardiologists from Poland who were heardly welcomed (Figure 4).

\section{Course structure}

The course contains 6 modules of 4 days of lectures, interactive sessions, live transmissions and case presentations by the participants over a 2 -year period.
The first module addressed the topic 'How to Approach Heart Failure'chaired by Bertram Pitt from Ann Arbor, Michigan and began with lectures from eminent cardiologists such as Martin Cowie and Theresa McDonagh from London as well as Christian Mueller from Basle, Switzerland (Figure 5). The importance and assessment of heart failure was reviewed. These topics were further pursued in Rapid Fire Sessions (Figure 6) and presentations of challenging cases by the participants themselves. An important subject was the pathophysiology of heart failure reviewed by John Cleland from Glasgow, the haemodynamic changes and changes in the cardiac marker structure in such patients reviewed by Stephan Rosenkranz from Cologne, Germany. 


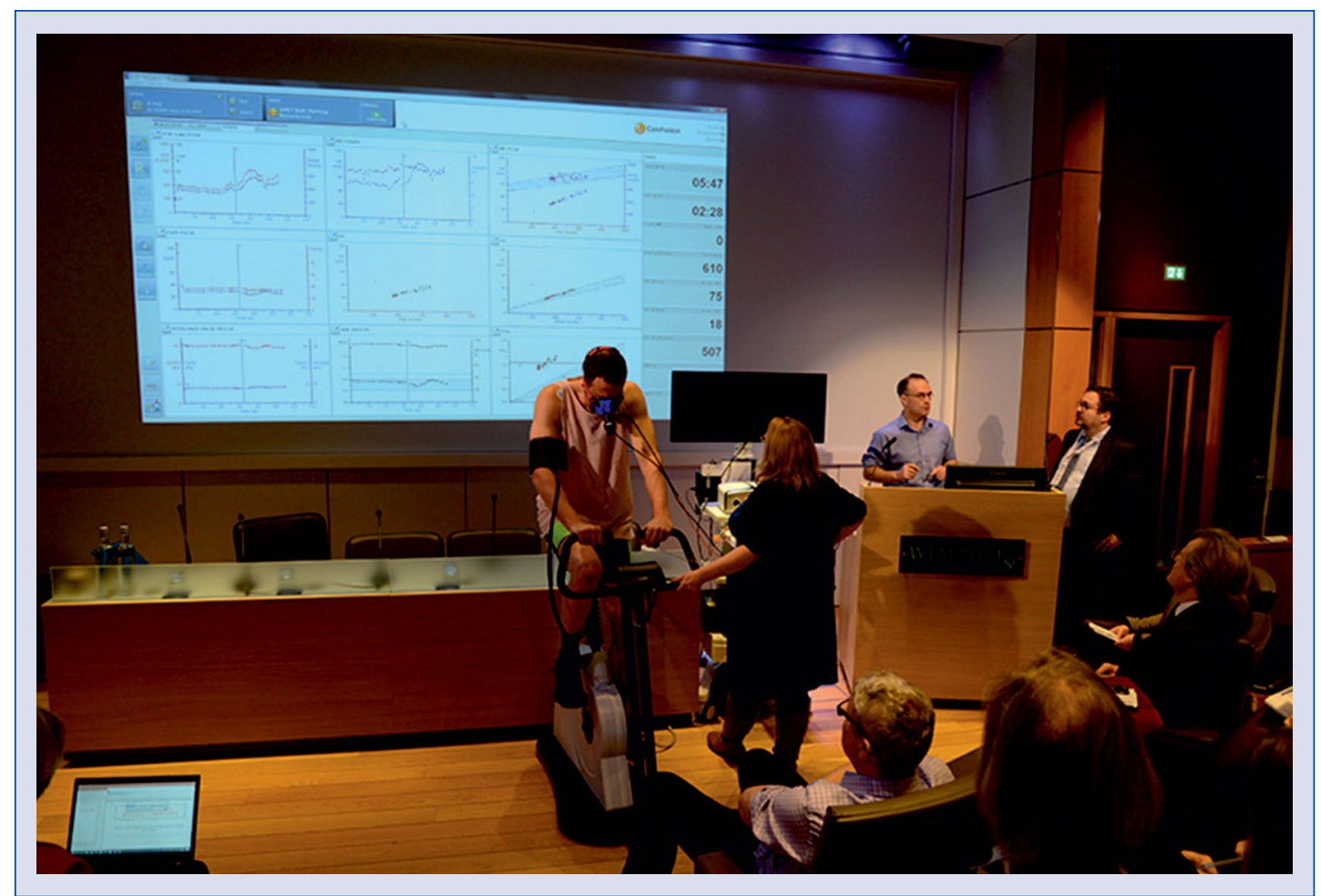

Figure 7. Live demonstration of an ergospirometry by Luke Howard and Daniel Dumitrescu (Credit J. Lipton).

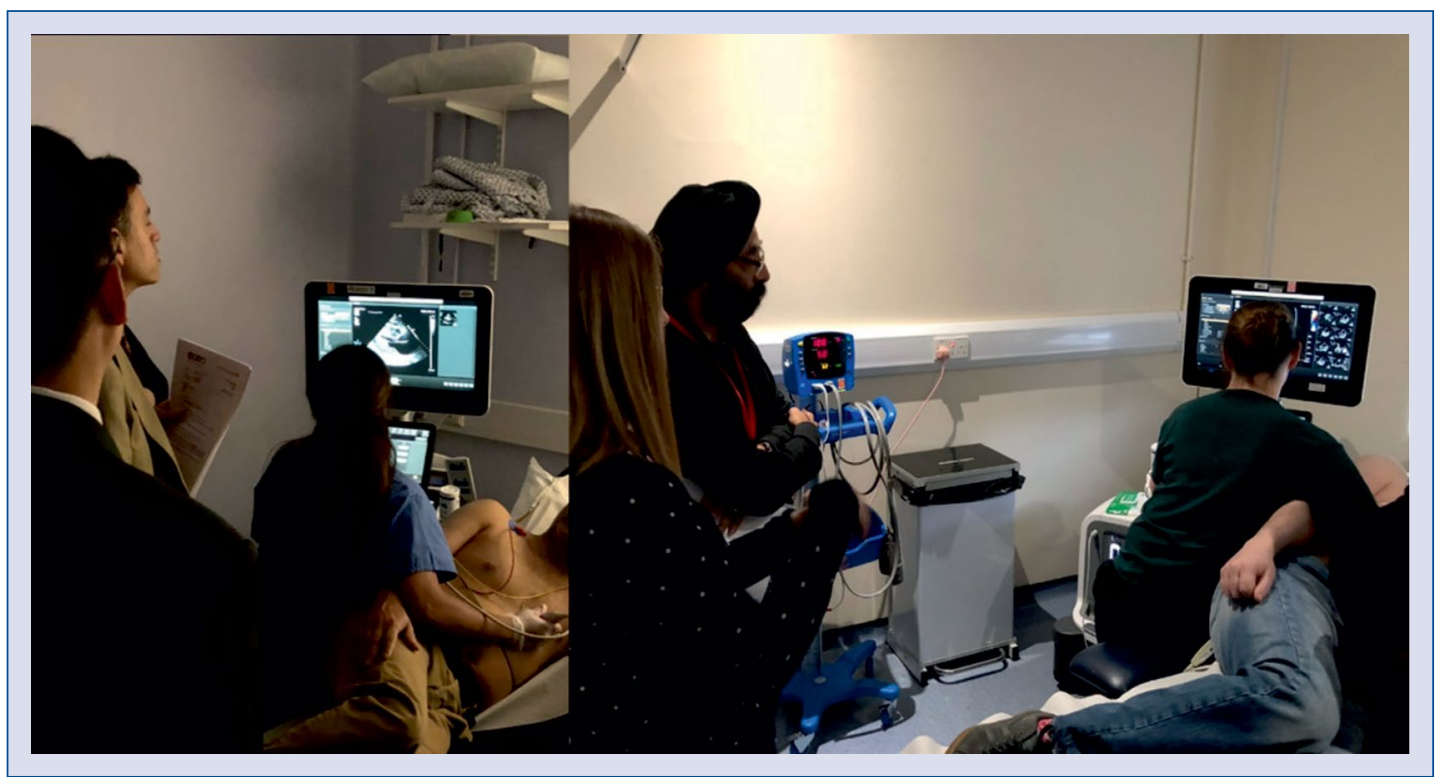

Figure 8. Hands-on echocardiography session at the Royal Brompton Hospital.

A highlight was a LIVE demonstration of an ergospirometry by both a pulmonologist and a cardiologist with an on-line transmission of the detailed oxygen metabolism as well as cardiohaemodynamic parameters (Figure 7). This was followed by another LIVE transmission by Roxy
Senior and his team of a stress echocardiography with contrast, which impressively demonstrated the potential of this examination, if performed at such a professional level.

The first module was further complemented by lectures on comorbidities in heart failure by Christian 
Angermann from Würzburg, Germany, diabetes and coronary artery disease and heart failure by Martin C. Petrie from Glasgow and finally on stunning and hibernation by Gerd Heusch from Essen, Germany.

Furthermore, diagnostic procedures such as left and right heart catheterisation and coronary angiography were demonstrated in impressive Live-in-a-box sessions by Stefan Rosenkranz and Ronald Binder from Wels, Austria.

For the assessment of patients with heart failure imaging technologies play a crucial role, as was outlined by highly educational lecture by Jeroen J. Bax, the former ESC President, on echocardiography and by Sanjay Prasad and Dudley
Pennel from London on cardiac magnetic resonance imaging and Ronald Büchel from Zurich, on hybrid imaging to guide revascularisation in heart failure.

The Saturday part of the course focused on congenital heart disease with eminent lectures by Michael Gatzoulis, Andrew Bolger and Sonya BabuNarayan followed by hands-on echo sessions, in small groups, at the Royal Brompton and Harefield Hospitals (Figure 8).

Thus, the London Postgraduate Course on Heart Failure started very successfully with happy participants and organizers and will continue in June with model 2, again at the Royal Society of Medicine in London. 